\title{
Memory Nanodevices Based on Carbon Nanotube-Fe-Pt Interconnects: Electromagnetic Simulations and Magnetically Stimulated Nanotube Growth
}

\author{
Stefano Bellucci ${ }^{1}$, Federico Micciulla ${ }^{1}$, Yuri Shunin ${ }^{2}$, Yuri Zhukovskii ${ }^{2}$, Viktor Gopejenko $^{3}$, Nataly Burlutskaya ${ }^{3}$, \\ Tamara Lobanova-Shunina ${ }^{4}$ and Aldo Capobianchi ${ }^{5}$ \\ 1. INFN - Laboratori Nazionali di Frascati, Via Enrico Fermi 40, I-00044, Frascati- (Rome), Italy \\ 2. Institute of Solid State Physics, University of Latvia, LV-1063 Riga, Latvia \\ 3. ISMA University, LV-1019 Riga, Latvia \\ 4. Aviation Institute, Riga Technical University, LV-1019 Riga, Latvia \\ 5. Istituto di Struttura della Materia-CNR A.d.R. Roma, Via Salaria Km 29.300 Monterotondo (Rome) Italy
}

\begin{abstract}
The parametrically controlled production of CNTs (Carbon nanotubes) with predefined morphologies is a topical technological problem for modern nanoelectronics. The CVD (Chemical vapor deposition) technique for SWCNTs (Single walled carbon nanotubes) in the presence of various metal nanoparticle catalysts is generally used now. The application of a magnetically stimulated CVD process scheme and catalyst nanoparticles with a strong magnetism promises additional possibilities for the CVD process management and allows expecting a predictable growth of CNTs with set chiralities and diameters. The main attention is focused on the magnetically anisotropy Pt-Fe in L10 crystallographic phase nanoparticles effect research. The developed theoretical cluster approach based on the multiple scattering and effective medium approximation is used for simulation of fundamental electromagnetic properties in Pt-Fe L10-CNT interconnects, which are responsible for developing CNTs morphologies. The proposed model of "effective bonds" and the model of magnetic stimulation for growing CNTs morphologies generated on the Pt-Fe nanoparticle surface are applied for the evaluation of the expected CNT chiralities distribution. The model and conditions controlled magnetically, which stimulate CNT growth in the CVD process, aimed at the predictable SWCNT diameter and chirality and based on Pt-Fe L10 catalyst are discussed. The possibilities of CNT forest growing on FePt nanoparticles for magnetic nanomemory are also evaluated.
\end{abstract}

Key words: CVD (Chemical vapor deposition), CNTs (Carbon nanotubes), magnetically controlled growth, arc discharge technique, Pt-Fe nanodrops-catalysts, fundamental electromagnetic properties, Pt-Fe L10 nanoparticles, interconnect, CNT growth modeling.

\section{Introduction}

CNTs (Carbon nanotubes) of various chiralities open new wide possibilities for modern nanoelectronics as promising candidates for nanointerconnects in a high-speed electronic nanosensoring and nanomemory devices [1-7].

We focus our current study on the implementation of advanced simulation models for a proper description of

\footnotetext{
*Corresponding author: Stefano. Bellucci, professor, first researcher, research fields: theoretical physics, condensed matter, nanoscience and nanotechnology, nanocarbon based composites, biomedical applications. E-mail: Stefano. Bellucci@lnf.infn.it.
}

the fundamental electromagnetic properties (electrical resistance, capacitances and impedances) in contacts between carbon nanotubes of different morphologies and metallic substrates of different nature. We also present the model of magnetically stimulated CNT growth for a special case of Fe-Pt metallic nanoparticles, which have unique magnetic properties. We expect that in the presence of magnetic field, the CNTs growth will be more determined from the point of view of possible CNTs morphologies. Moreover, the creation of a CNT forest based on Pt-Fe nanoparticles provides the possibilities to consider this kind of structure as the basic fragment of nanomemory devices, 
where information bits are located in nanoparticles and the CNT forest provides the necessary spin transport for reading and recording information.

Thus, in our simulations, we expect to reproduce not only a CNT forest with the predefined morphology but also to develop a prototype of a nanomemory device.

The adequate description of CNT chirality [2] is one of the key points for a proper simulation on electric properties of CNT-based nanoelectronic devices.

The further development of the cluster approach allowed us to formulate the "effective bonds" model [5] and to carry out a cycle of simulations on electromagnetic properties in various CNTs- and graphene (GNRs- graphene nanoribbons)- based metal interconnects $(\mathrm{Me}=\mathrm{Fe}, \mathrm{Cu}, \mathrm{Ag}, \mathrm{Pt}, \mathrm{Au}, \mathrm{Pd}, \mathrm{Ni})[3,8]$. We consider that this model serves as a tool for understanding the process of CNT growth adequate to CVD (Chemical vapour deposition) process.

The main goal of the current research is to understand if there is a relationship between the use of magnetic catalysts and the CVD growth of CNTs, taking into account that most commonly used materials for the growth of CNTs are just $\mathrm{Fe}, \mathrm{Co}$ and $\mathrm{Ni}$ nanoparticles. The nanoparticles of these catalysts are magnetically isotropic. The key question arises: What would happen if one used instead of $\mathrm{Fe}, \mathrm{Co}$, and $\mathrm{Ni}$ nanoparticles, magnetically anisotropic nanoparticles such as those in the alloy Fe-Pt? The anisotropy of $\mathrm{Fe}-\mathrm{Pt}$ alloy is due to a spin-orbit coupling of $\mathrm{Fe}$ and $\mathrm{Pt}$ orbitals. This coupling takes place only if the alloy is formed of alternating planes of $\mathrm{Fe}$ and $\mathrm{Pt}$. This particular structure is called L10. In this way, because of the different sizes of the atoms of $\mathrm{Fe}$ and $\mathrm{Pt}$, the structure is crushed and atoms of $\mathrm{Fe}$ and $\mathrm{Pt}$ are close forming a centred tetragonal phase $(f c t)$. This approchement allows for the coupling and then the magnetic anisotropy with the magnetization axis perpendicular to such planes. It is essential in this case to find conditions to control the growing CNT chirality. We also should take into account a possible substitutional disorder of $\mathrm{Fe}_{\mathrm{x}} \mathrm{Pt}_{1-\mathrm{x}}$ alloy, when the stoichiometry number $\mathrm{x}$ becomes an additional parameter of CNT growth. No doubt, the diameter of the grown CNT is also an essential parameter, which is evidently pre-defined by the created nanoparticle diameter. But again, we should discuss some limitations in the creation of Fe-Pt nanoparticle sizes in connection with the Fe-Pt melting point. However, Fe-Pt nanoparticles demonstrate an extremely strong coercing field (about, for the phase $f c t$ in some special compositions and this promises definite hopes for the controlled CNT growth in the CVD process. We also consider the CVD process of CNT growth as a more predictable one from the point of view of the expected growing CNT parameters (diameter, chirality, morphology of SW or MW CNTs)).

Our experience with the nanotubes synthesis by means of the arc discharge brought about large doubts in the possibilities of CNTs growth control. The synthesis was carried out according to the parameters presented here: Current $90 \mathrm{~A}$, Voltage $\sim 18 \div 20 \mathrm{~V} \mathrm{DC}$, Vacuum $\sim 2 \times 10^{-4}$ mbar, Gas: He, Work Pressure 600 mbar, arc duration $\sim 15 \mathrm{~min}$.

Some filaments also exit from the outer wall of the cathode but in smaller quantities than found on the anode. The residue of the deposit formed on the upper part of the synthesis chamber was collected. It appeared to be quite little compared to other discharges with other catalyst (Figs. 1 and 2).

The Fig. 2 shows that filaments are of different nature - some formats are created from these spheres and others have very fine structures of a few nanometers, probably single-walled nanotubes, covered by these spheres, partly or totally. The question is: What percentage of nanoparticles was originally made or how much iron and platinum and how this underwent a change, during the arc discharge process?

We also take into account the extremely marginal parameters of the Pt-Fe system: Pt has the melting point at $\mathrm{T}=2,041.4 \mathrm{~K}\left(1,768.2{ }^{\circ} \mathrm{C}\right)$ and the boiling point at $\mathrm{T}=4,098 \mathrm{~K}\left(3,825^{\circ} \mathrm{C}\right)$; 


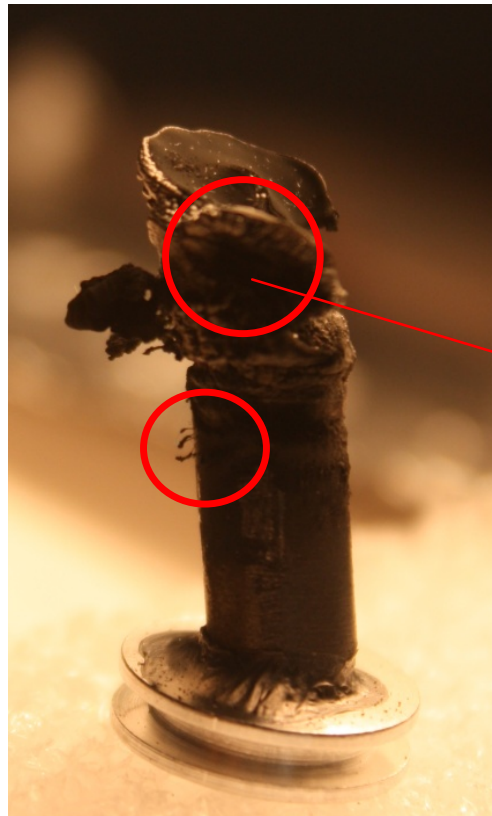

(a)

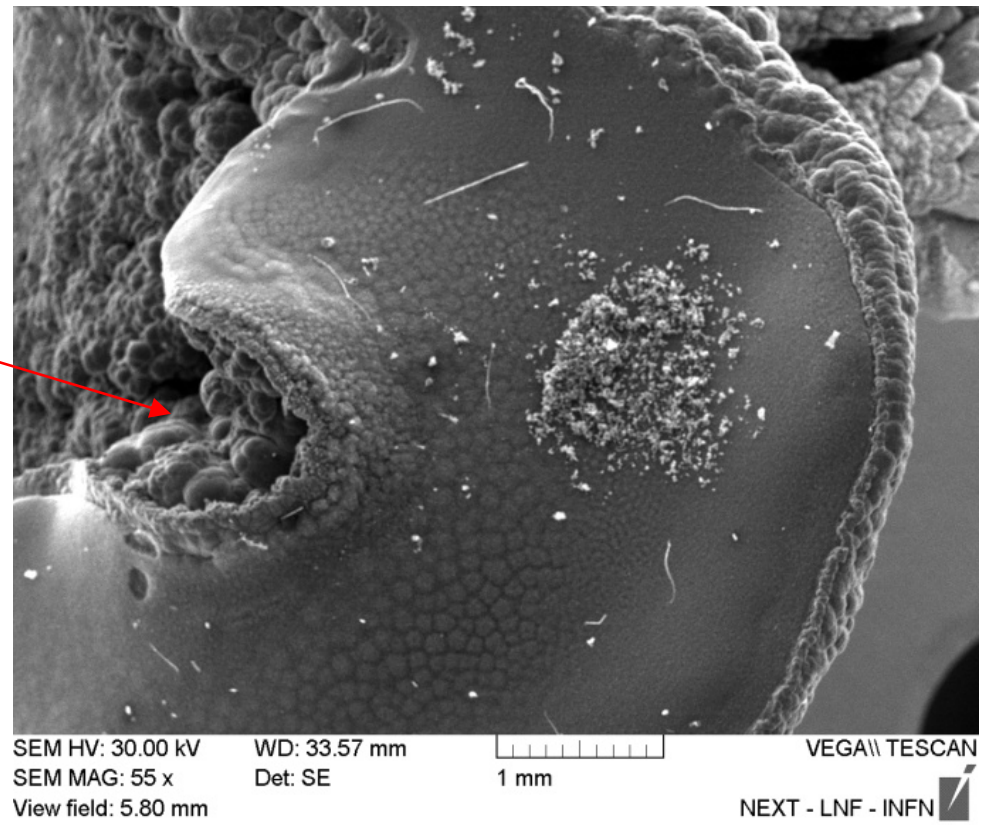

(b)

Fig. 1 (a) Photo of the cathode filaments exiting out after the synthesis; (b) SEM photo of the cathode surface.

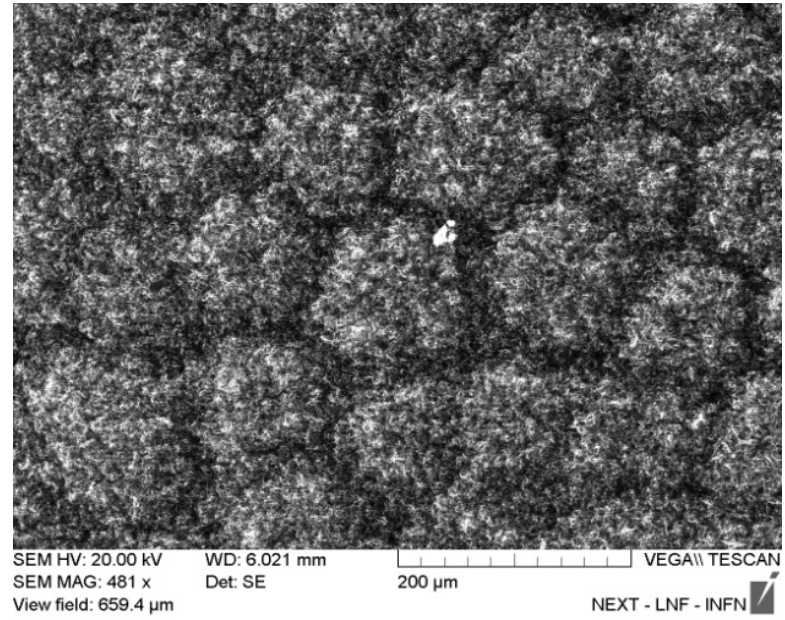

(a)

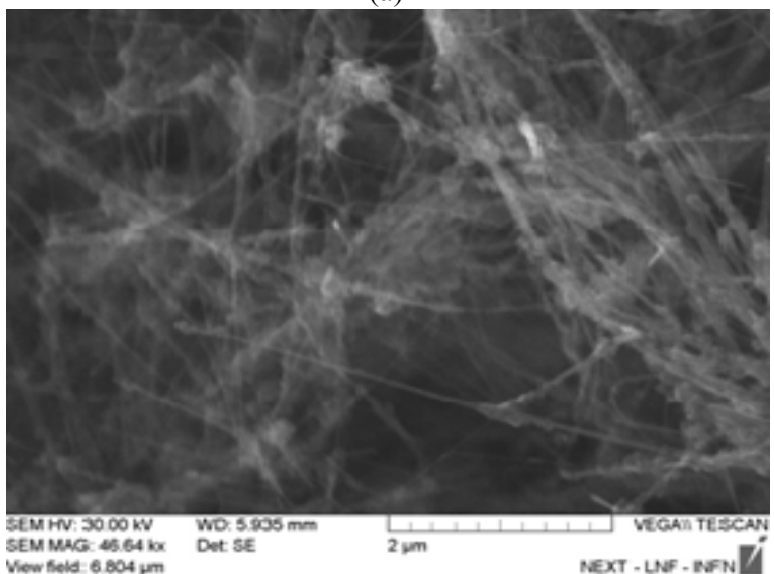

(c)

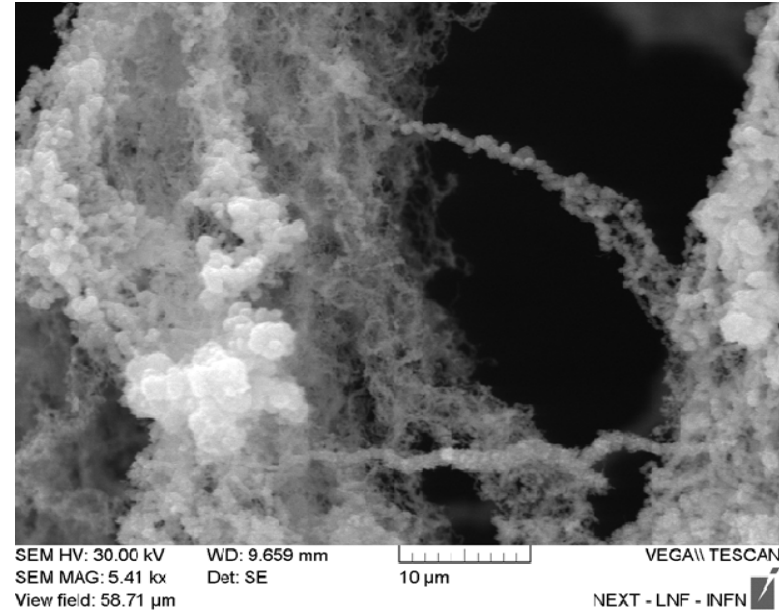

(b)

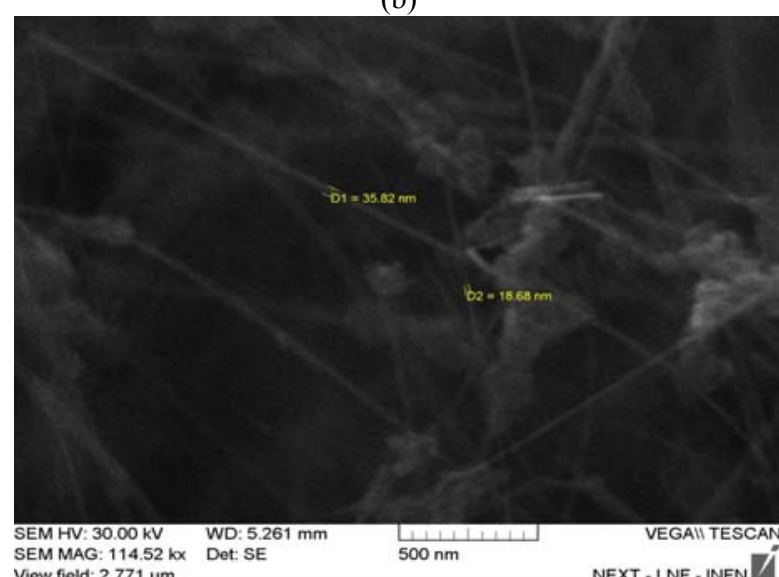

(d)

Fig. 2 Evolution view of filaments in the arc discharge process (Bellucci-Capobianchi): (a), (b), (c) and (d) [9-12]. 
There are four major crystal structure modifications for Fe: below $769{ }^{\circ} \mathrm{C}$ (Curie temperature) it is $\alpha$-Fe $(\alpha$-ferrite) with a body-centered cubic crystal structure and ferromagnetic properties. Ferrite above the critical temperature $\left(769-917^{\circ} \mathrm{C}\right)$ is beta-ferrite $(\beta-\mathrm{Fe})$ where it is paramagnetic rather than ferromagnetic and it is crystallographically identical to $\alpha$-Fe. Within the interval of $917-1,394{ }^{\circ} \mathrm{C}$ it is $\gamma$-Fe (austenite) with acicular cubic crystal structure. At temperatures between $1,394{ }^{\circ} \mathrm{C}$ and $1,538{ }^{\circ} \mathrm{C}$, the body-centered cubic crystal structure is the more stable form of delta-ferrite $\left(\delta\right.$-Fe), the melting point $1,538{ }^{\circ} \mathrm{C}(1,811 \mathrm{~K})$, and the boiling point at $\mathrm{T}=3,273 \mathrm{~K}\left(3,000{ }^{\circ} \mathrm{C}\right)$.

Our earlier research proved that close to the arc the temperature ranges between $4,000-6,000 \mathrm{~K}$ and there is a subsequent rapid temperature gradient decrease with the distance from it. Filaments are found nearby the arc itself, more precisely, upstream of it. Taking into account the essentially non-steady character of the arc discharge process and very high working temperatures, the CNT growth control is practically impossible and CNT morphologies are non-predictable.

\section{Deposition Process Based on Metal Nanoparticles}

The CVD is a highly versatile approach to producing nanotubes and is, perhaps, the most commonly published technique for nanotube growth often used to synthesize CNTs for commercial applications. The process involves decomposition of a carbonaceous precursor at high temperatures under oxygen-free conditions and reduced atmosphere to produce nanotubes.

The substrate needs to have catalyst nanoparticles providing the growth of CNTs. Nanoparticles can be composed of lots of different substances (usually, a metal like Fe, Co, Ni Co, Mo, Mn, Pd etc.) [13]. Catalyst metals mostly used for these purposes are listed in the following Table 1.

It is necessary to emphasize that it will be possible to grow CNTs using a gas, both as a catalyst and as hydrocarbon. This approach yields MWNTs, whereas with gas-phase pyrolysis of acetylene using a metallocene yields SWNTs with diameters around $1 \mathrm{~nm}$ [15].

\section{CNTs Growth in the Chemical Vapor}

Table 1 Most commonly used catalyst metals for the CVD method [14].

\begin{tabular}{lllll}
\hline Catalyst & & & Temperature, C & Carbon source \\
\hline Metal & Catalyst type & Preparation method & & Benzene \\
$\mathrm{Fe}$ & Ultra fine particle & Decomposition of metallocene & 1,060 & Acetylene \\
& Silica support & Pore impregnation & 700 & Acetylene \\
& Zeolite or clay support & Ion exchange & 700 & Acetylene \\
& Graphite support & Impregnation & 700 & Acetylene \\
& Ultra fine particle & Decomposition metal of carbonyl & 800 & Acetylene \\
& Silica support & Sol-gel process & 700 & Triazine \\
$\mathrm{Co}$ & Ultra fine particle & Laser etching of Co thin film & 1,000 & Acetylene \\
& Ultra fine particle & Decomposition metal of carbonyl & 800 & Acetylene \\
& Silica support & Pore impregnation & 700 & Acetylene \\
& Zeolite or clay support & Ion exchange & 700 & Acetylene \\
& Graphite support & Impregnation & 700 & Acetylene \\
$\mathrm{Ni}$ & Graphite support & Impregnation & 700 & Acetylene \\
& Ultra fine particle & Decomposition of $\left.\mathrm{Ni}_{8} \mathrm{H}_{12}\right)_{2}$ & 800 & Acetylene \\
$\mathrm{Mo}$ & Ultra fine particle & Decomposition of $\mathrm{Mo}{ }^{* 1}$ & 800 & Acetylene \\
$\mathrm{Mn}$ & Ultra fine particle & Decomposition metal of carbonyl & 800 & Acetylene
\end{tabular}

${ }^{1} \mathrm{Mo}^{*}=\left(\mathrm{NH}_{4}\right)_{25} \pm 5\left[\mathrm{Mo}_{154}(\mathrm{NO})_{14} \mathrm{O}_{420}(\mathrm{OH})_{28}\left(\mathrm{H}_{2} \mathrm{O}\right)_{70}\right] \cdot 350 \cdot \mathrm{H}_{2} \mathrm{O}$ 
CVD-produced CNTs are curved and have high amount of defects, mainly after the purification in the acid bath; it is needed to remove metal nanoparticles inside of tubes.

The main interests related to iron-containing nanoparticles are focused on their potential applications as high-quality magnetic materials. Thus, a new generation of iron-metal nanoparticles is studied to be used as a catalyst in growing CNTs, with a magnetic nanomaterial inside, making it possible to design a precise carbon nanomagnetic device for drug delivery diagnostics. For example, iron nanoparticles have been widely used as a catalyst for CVD synthesis of multiwalled carbon nanotubes [16], while iron-molybdenum can act as a very efficient catalyst for the synthesis of either single-walled or multiwalled carbon nanotubes with the CVD method [17-22]. To prevent the magnetic behaviour of these iron-nano-compounds, it is necessary to grow carbon nanotubes of nanocompounds below the Curie temperature. Typically, the growth temperature is around $600-700{ }^{\circ} \mathrm{C}$ at the atmospheric pressure [23]. The potential applications of carbon nanotubes grown for semiconductor and sensor devices are presented for CMOS industrial applications [24]. The low-temperature growth of vertically aligned CNTs at high growth rates by a PTCVD (Photo-thermal chemical vapour deposition) technique using a $\mathrm{Ti} / \mathrm{Fe}$ bilayer film as a catalyst is presented in reference [25]. The bulk growth temperature of the substrate is as low as $370{ }^{\circ} \mathrm{C}$ and the growth rate is up to $1.3 \mu \mathrm{m} / \mathrm{min}$, at least eight times faster than the values reported by traditional thermal CVD methods.
It should be recognized that the mechanism of CNTs growth is not obvious enough. Technological growth characteristics may differ in detail, although the concept remains the same. The mechanism of carbon atoms deposition on metal catalyst nanoparticles with the subsequent nucleation of CNTs can be considered one of the most effective and practically important. Crystal (eg. Si) nanoparticles with a set diameter created on a substrate give a high probability of producing CNTs with the regulated diameter. However, the problem of controlling the chirality of CNTs remains a pressing one. Among more effective catalysts, it is possible to distinguish $\mathrm{Pt}$, $\mathrm{Pd}$, $\mathrm{Cu}, \mathrm{Ag}, \mathrm{Au}, \mathrm{Si}, \mathrm{SiC}, \mathrm{Ge}, \mathrm{Al}_{2} \mathrm{O}_{3}[25]$ in addition to the earlier investigated $\mathrm{Mg}, \mathrm{Ti}, \mathrm{Cr}, \mathrm{Mn}, \mathrm{Fe}, \mathrm{Co}, \mathrm{Ni}, \mathrm{Sn}, \mathrm{Pb}$ [27-29].

\section{Magnetically Stimulated CNTs CVD Growth on Fe-Pt Catalysts}

\subsection{Effective Bonds Model for Cnt-Pt-Fe Interconnects Electromagnetic Properties}

We have developed structural models for CNT-Me and GNR-Me junctions, based on their precise atomistic structures - clusters, which take into account the CNT chirality effect and its influence on the interconnect resistance for $\mathrm{Me}(=\mathrm{Fe}, \mathrm{Ni}, \mathrm{Cu}, \mathrm{Ag}, \mathrm{Pd}, \mathrm{Pt}, \mathrm{Au})$ as well as the pre-defined CNT (or GNR) geometry. These atomistic structures are in compliance with the proposed "effective bonds" model. The "effective bonds" are responsible for mechanical, electronic, magnetic and electrical properties of interconnects. The common consideration of two marginal carbon structures

Table 2 Comparison of catalysts for SWCNTs growth [27-29].

\begin{tabular}{lllllll}
\hline Type & State & $\begin{array}{l}\text { Size for SWCNT } \\
\text { growth }\end{array}$ & $\begin{array}{l}\text { Catalysis for } \\
\text { cracking }\end{array}$ & Carbon solubility & $\begin{array}{l}\text { Growth temperature } \\
\text { for ethanol ( }{ }^{\circ} \text { ) }\end{array}$ & Growth rate \\
\hline $\mathrm{Fe}, \mathrm{Co}, \mathrm{Ni}$ & Liquid/solid & $<10 \mathrm{~nm}$ & Yes & High & $600-950$ & High \\
$\mathrm{Pt}, \mathrm{Pd}$ & Liguid/ solid & $<5 \mathrm{~nm}$ & No? & High & $850-950$ & High \\
$\mathrm{Au}, \mathrm{Ag}, \mathrm{Cu}$ & Liquid/solid & $<5 \mathrm{~nm}$ & No & Low & $850-950$ & High \\
$\mathrm{Ge}$ & Liquid/solid & $<5 \mathrm{~nm}$ & No & Low? & $850-950$ & Low? \\
$\mathrm{Si}, \mathrm{SiC}$ & Solid & $<5 \mathrm{~nm}$ & No & No? & $850-950$ & Low \\
$\mathrm{Al}_{2} \mathrm{O}_{3}$ & Solid & $<5 \mathrm{~nm}$ & No & No & $850-950$ & Low \\
\hline
\end{tabular}


(CNTs and GNRs) is induced by the similar technological problems in respect of these materials for the modern nanoelectronics.

Our main interest in this particular case is focused entirely on CNT-Me interconnects, when the modelling of CNTs growth on metal surfaces is combined with the controlled electromagnetic properties in the interconnect area. To ensure a predictable interconnect morphology, the permanent magnetic field is used that accompanies the growth, and magnetic drops are used as growth catalysts that provide for the special composition of Fe-Pt with unique magnetic properties. The results of our simulations show that interconnects resistance and the number of effective bonds can be considered as indicators of chirality. Figs. 3-7 demonstrate the numbers of effective bonds via CNT diameters, chirality angles, CNT-Me interconnects resistances and impedances. It means that resistances and the number of effective bonds in the interconnect space are indicators of CNTs morphology.

\subsection{CNT-Fe Pt $_{1-x}$ Interconnect Formation}

There is a relation between the use of magnetic catalysts and the CVD growth of CNTs determining the

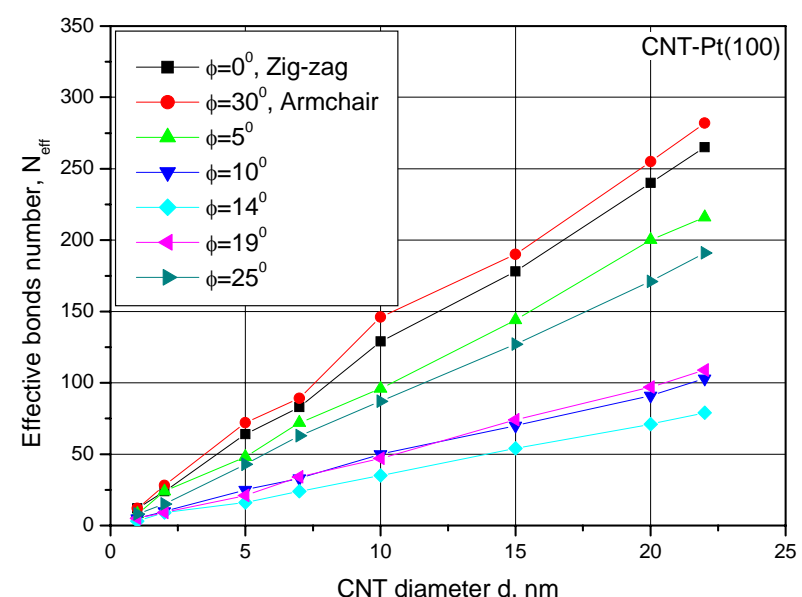

(a)

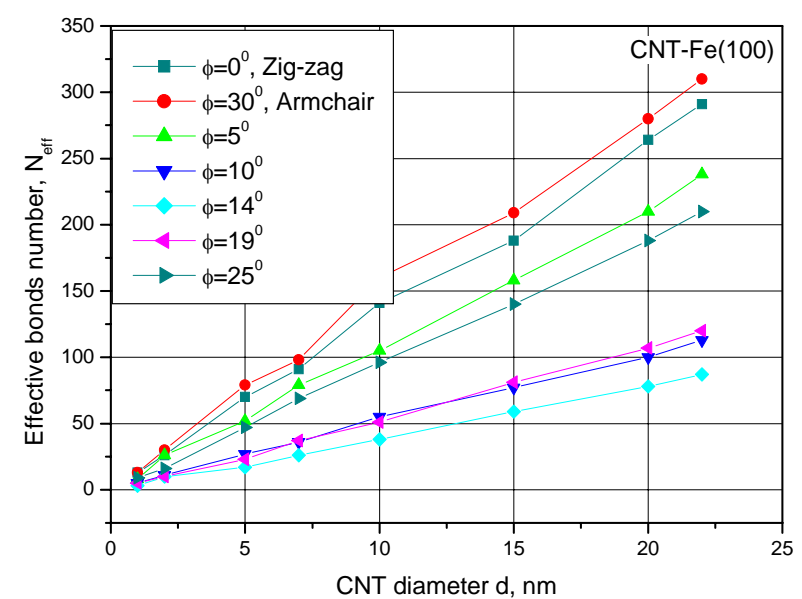

(b)

Fig. 3 Effective bonds number via CNT diameter: (a) CNT-Pt and (b) CNT-Fe. 

Electromagnetic Simulations and Magnetically Stimulated Nanotube Growth

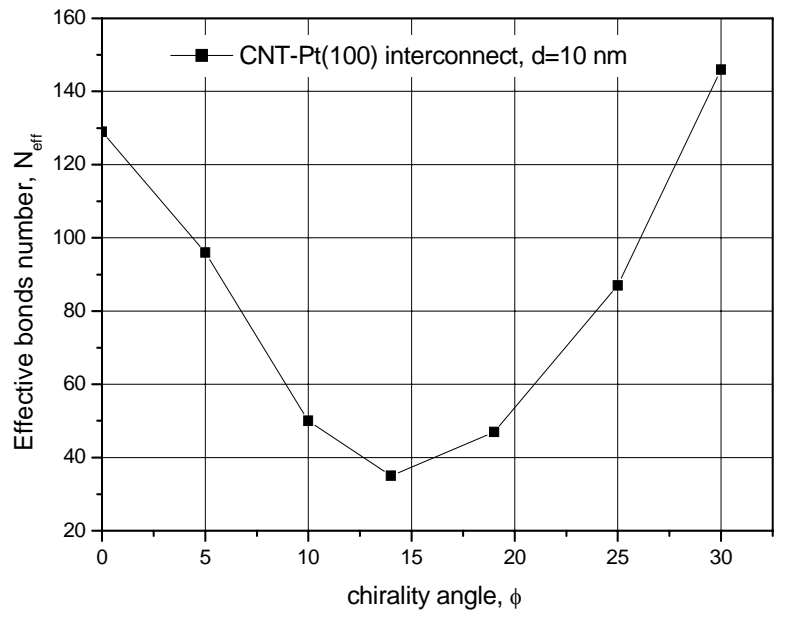

(a)

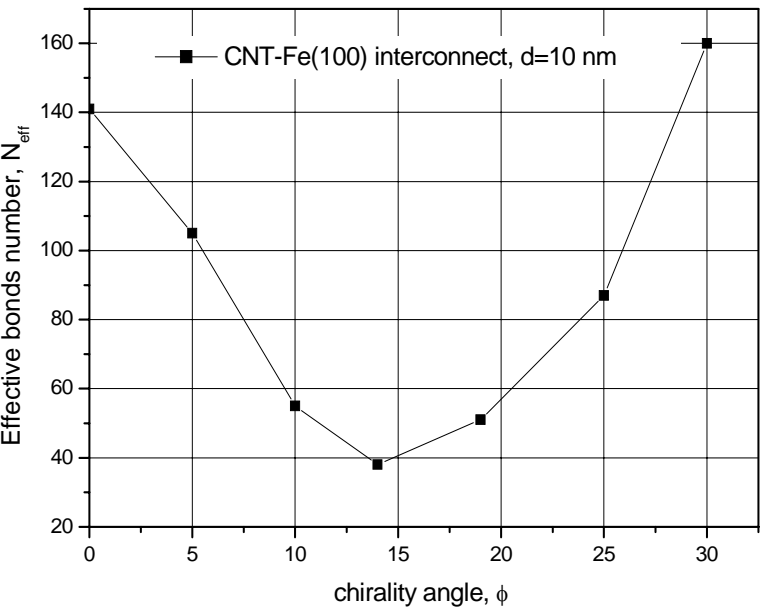

(b)

Fig. 4 Effective bonds number via CNT chirality for the diameter $\mathrm{d}=10 \mathrm{~nm}$ : (a) CNT-Pt and (b) CNT-Fe.

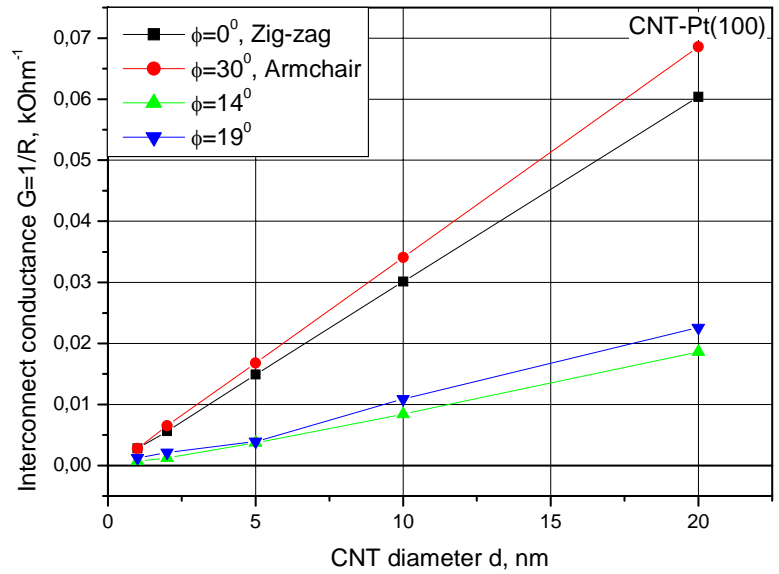

(a)

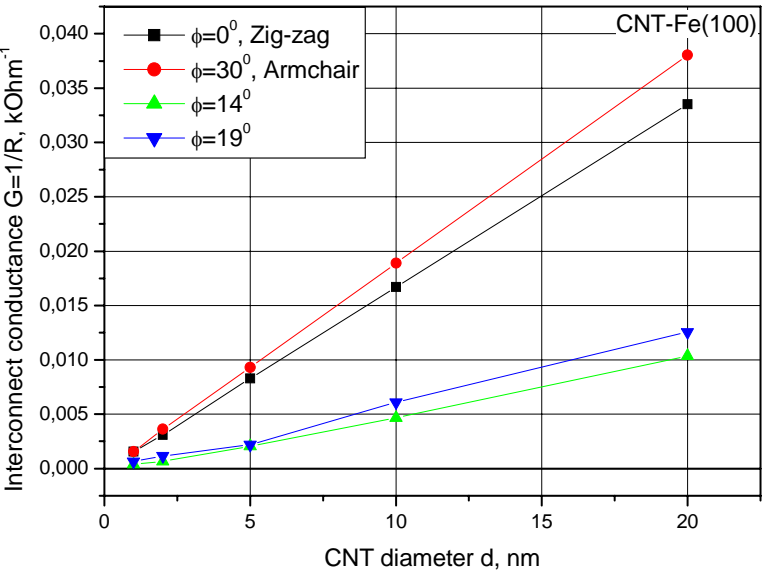

(b)

Fig. 5 Interconnect conductance via CNT diameter: (a) CNT-Pt and (b) CNT-Fe.

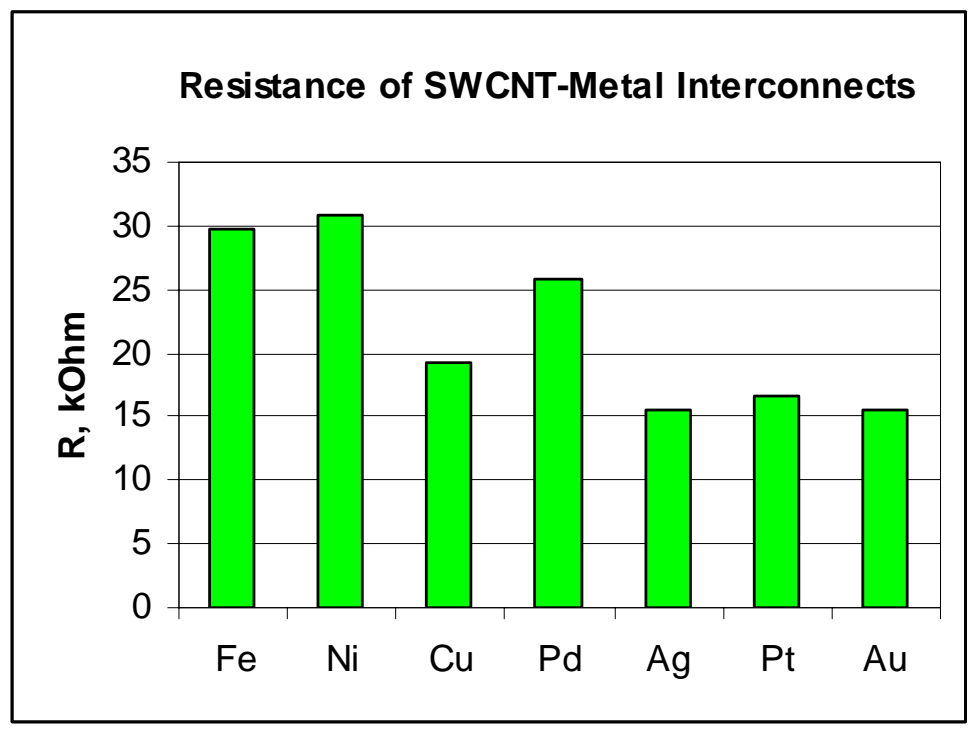

Fig. 6 Resistances of zig-zag SWCNT-Metal interconnects in comparison. 


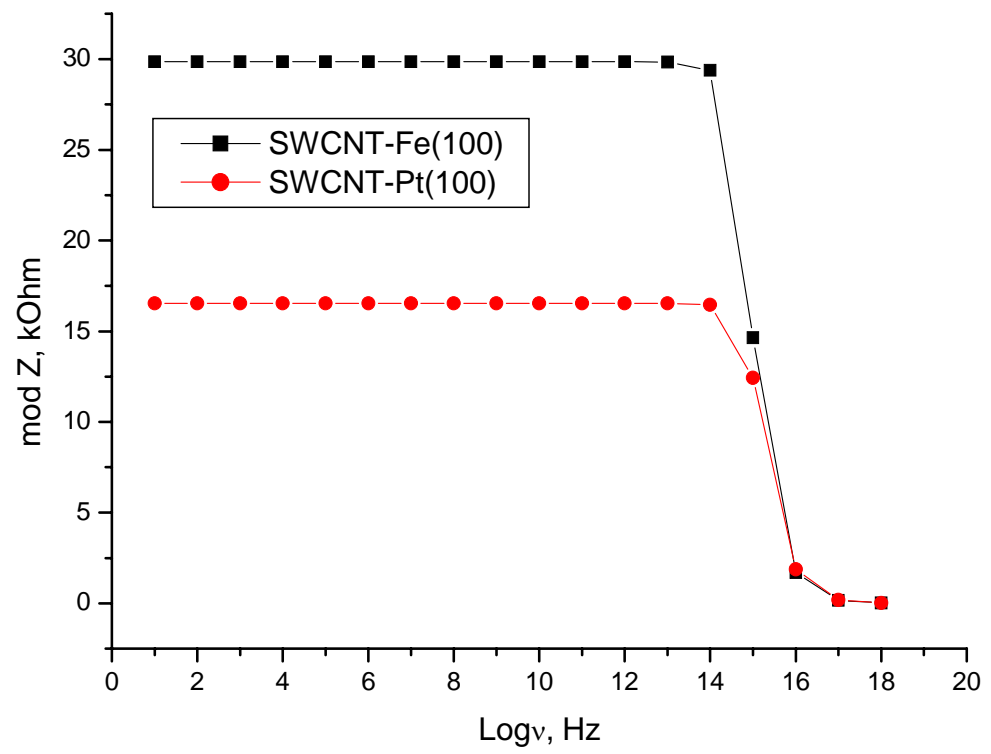

Fig. 7 Impedances of SWCNT-Fe and -Pt interconnects in comparison.

\subsubsection{Magnetic Properties of Fe-Pt Alloys.}

The unique magnetic properties of Fe-Pt alloys - is an open field for research to correlate different CNT techniques in terms of the catalyst role in entirely different range of temperatures and pressures. Carbon nanotubes grow using bimetallic nanoparticles Fe-Pt as a catalyst [31-34]. Since the mid-1930s Fe-Pt alloys have been known to exhibit high coercivities due to high magneto crystalline anisotropy of the $\mathrm{L} 1_{0} \mathrm{Fe}-\mathrm{Pt}$ phase but their high cost prevented these alloys from widespread applications in the past. In Fe-Pt alloys, both Fe and Pt atoms carry a magnetic moment: The induced magnetic moment on the $\mathrm{Pt}$ sites and the enhanced magnetic moment on the Fe sites. A wide variety of the magnetic structure types in the Fe-Pt alloys is evidently the consequence of various atomic configurations around $\mathrm{Fe}$ atoms, which, in turn, has a considerable effect on the electronic structure of these alloys (both Table 3 and Eutetic Phase Diagram of Fe-Pt alloy (http://www.himikatus.ru/art/phase-diagr1/Fe-Pt.php)).

To obtain the Fe-Pt alloy in $\mathrm{L}_{0}$ phase, we use a new chemical synthesis strategy reported in details in our previous works [31-34]. By planetary ball milling of the nanocrystals of a particular precursor $\left[\mathrm{Fe}\left(\mathrm{H}_{2} \mathrm{O}\right)_{6}\right] \cdot \mathrm{PtCl}_{6}$ and $\mathrm{NaCl}$ were grounded and then annealing at $400{ }^{\circ} \mathrm{C}\left(300{ }^{\circ} \mathrm{C}\right.$ less than typical used temperature) in reductive atmosphere, After washing we obtain Fe-Pt $\mathrm{L1}_{0}$ NPs with selected size as function of fraction of $\left[\mathrm{Fe}\left(\mathrm{H}_{2} \mathrm{O}\right)_{6}\right] \cdot \mathrm{PtCl}_{6}$ and $\mathrm{NaCl}$ used. By varying the precursor ratio we are able to avoid the coalescence phenomena and obtain single crystal NPs with the size around $6 \mathrm{~nm}$, not agglomerated.

Table 3 Specific magnetic structures of Pt-Fe systems.

\begin{tabular}{lll}
\hline Magnetic structure & Behaviour & Curie temperature \\
\hline $\mathrm{L}_{2} \mathrm{Fe}_{3} \mathrm{Pt}$ & Ferromagnetic & $410 \mathrm{~K}$ \\
$\mathrm{~L}_{0} \mathrm{FePt}$ & Ferromagnetic & $750 \mathrm{~K}$ \\
$\mathrm{~L}_{2} \mathrm{FePt}_{3}$ & Paramagnetic & $273 \mathrm{~K}$ \\
\hline
\end{tabular}

3.2.2 Magnetically Stimulated CNTs Growth with the Probabilistically Pre-Defined Morphology

The formation of the initial optimal perimeter for C-Metal (Fe-Pt) bonds is a synergetic process with a minimal free energy (Fig. 8).

The nanoparticle diameter determines with a certain error the diameter of a CNT. The number of effective bonds defines the morphology of the future CNT (Fig. 9, arm-chair, chiral, zig-zag CNTs,) in terms of chirality. Obviously, there is a considerable uncertainty in the morphology of the future CNTs, owing to sporadic thermal dynamics of the deposited carbon atoms.

According to our model, the process can be considerably streamlined using even minor 
diamagnetic properties of carbon atoms at the expense

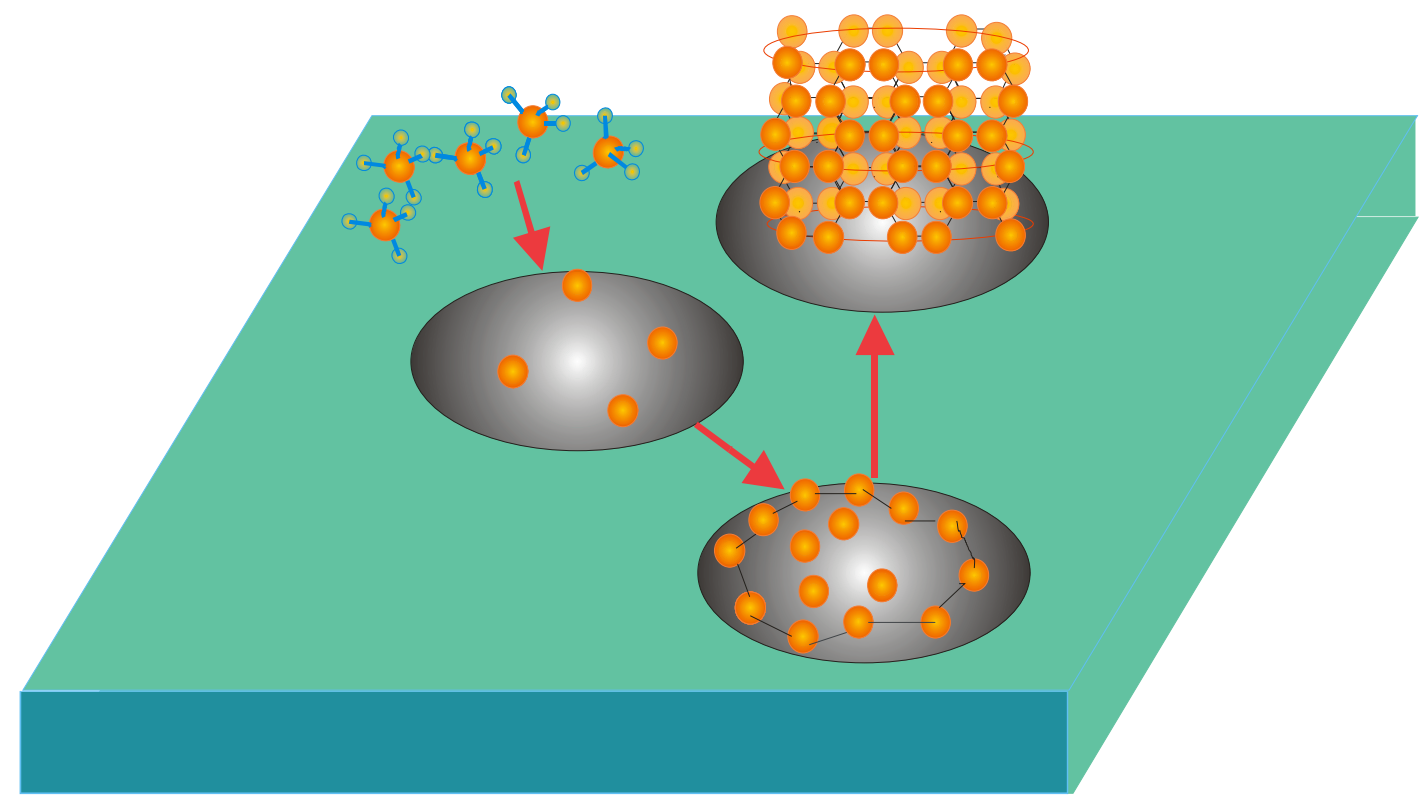

Fig. 8 A fragment of the CNT CVD process growth on nanoparticle surfaces.
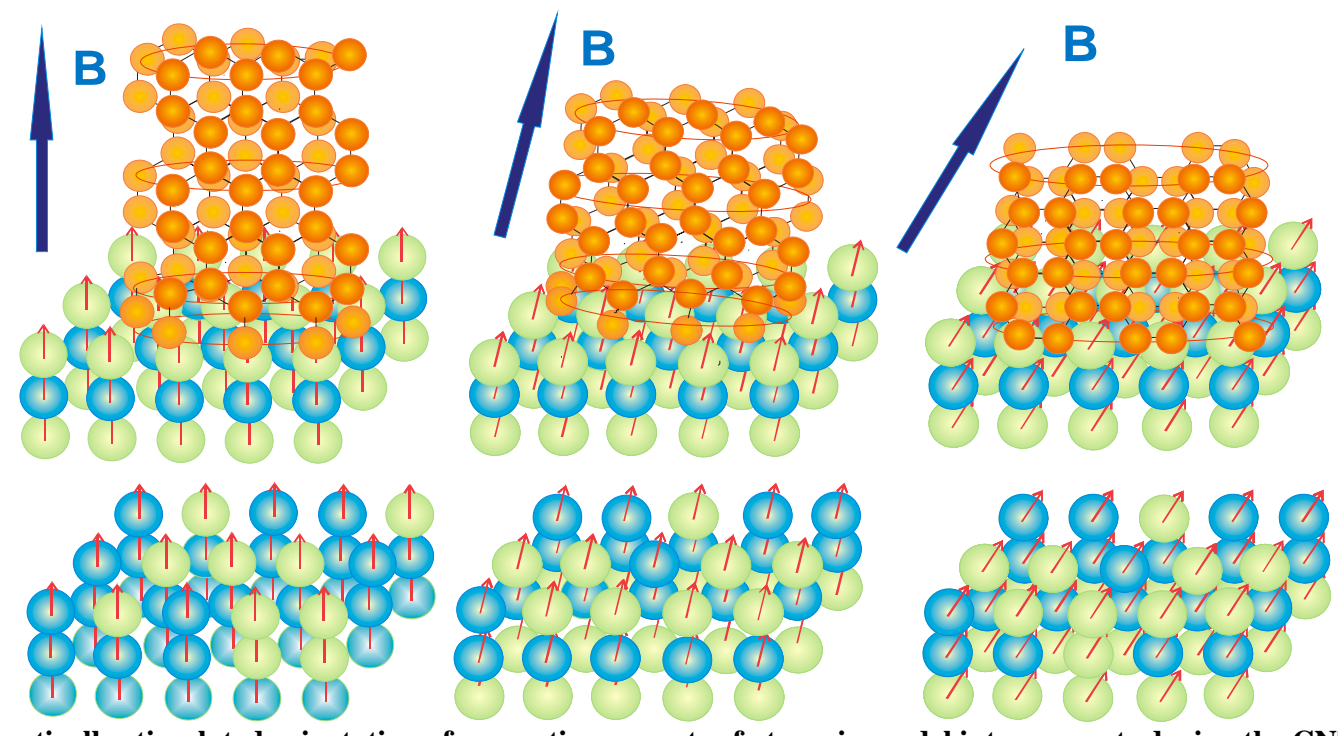

Fig. 9 Magnetically stimulated orientation of magnetic moments of atoms in model interconnects during the CNTs controlled growth with the expected chiralities under the directed mag $\phi=\Theta_{B}$ : (a) arm-chair CNT, magnetic field B with the orientation angle $\Theta_{B}$, where $\mathrm{B}$ is the magnetic induction and the chirality angle is $\phi=0^{\circ}$, (b) chiral $\mathrm{CNT}, 0<\phi<30^{\circ}$ and (c) zig-zag CNT, $\phi=30^{\circ}$.

of magnetic field and strong induced ferromagnetism of the nanoparticle. It becomes evident that the nanoparticle composition Fe-Pt and its atomic structure (including the short-range order) play a primary role in the process of CNT growth stimulated by the magnetic field of CVD.

Nucleation in the process of CNT growth in cases of the ordered Fe-Pt nanoparticles is more stable and has principal advantages in relation to the controlled CNTs growth compared with the cases of any kind of anisotropic Fe-Pt nanocatalysts [35].

The availability of substitutional disorder and anisotropy in Fe-Pt nanodrops used for CNT CVD controlled growth is a negative factor for the 
production of CNTs with predictable morphologies. There are some essential negative features of disorder in Fe-Pt nanodrops for the CVD process:

$\mathrm{C}$ atoms chemical binding preferences $(\mathrm{C}-\mathrm{Fe}$ bonds are stronger than C-Pt ones);

A higher structural anisotropy leads to higher relaxation times of a nanodrop structure [36];

Disordering effects in Fe-Pt lead to sporadic magnetization phenomena.

Thus, it is important to control the process of formation of nanodrop structures corresponding to $\mathrm{L} 1_{0}$ (Fe-Pt) and $\mathrm{L}_{2} \quad\left(\mathrm{Fe}_{3} \mathrm{Pt}\right)$ systems with reliable magnetism and adequate cooling rates, when relaxation processes in nanodrops are accomplished.

\subsubsection{Model of CVD CNT Growth}

Magnetically stimulated CNT CVD model formulation looks as follows (Figs. 8 and 9):

Effective bonds are responsible for the future chirality of CNT in the CVD processes.

The permanent magnetic field provides the alignment of CVD growth.

The binding with the Fe-Pt nanoparticle has a probabilistic character; the probability of binding of $\mathrm{C}$ atoms with $\mathrm{Fe}$ atoms is preferable as compared to $\mathrm{Pt}$ atoms.

The composition of Fe-Pt atoms in a nanoparticle and the atomic structure ordering are essential for chirality of the obtained CNTs.

The diameter of the future CNT is correlated with the nanoparticle size.

The direction of the magnetic field stabilizes the direction of CNT forest growth and is a tool of the growth control.

The ordering of a nanoparticle atomic structure, as well as a substitutional disorder or arbitrary disorder of a nanoparticle, is responsible for the magnetic state of the nanoparticle, which is essential for the possible future nanomemory devices.

Even a very strong magnetic field is not able to suppress thermal fluctuations of magnetic moments in carbon atoms. It is a different thing, when carbon diamagnetic atoms appear on the surface of a catalyst in the ferromagnetic condition where magnetism induced by the external field can be very strong and is able to correct the behaviour of carbon atoms by suppressing thermal fluctuations.

At the same time, the growth control over chiral and non-chiral nanotubes essentially depends on stoichiometric composition of Pt-Fe nanoparticles. The beginning of the nucleation process with the growth of nanotubes might be connected with stochastic fluctuations of the magnetic moment in a carbon atom relative to the direction of the local magnetic field in a nanoparticle. Distribution of the fluctuation angle obeys the Gaussian law:

$$
f(\theta)=\frac{1}{\sigma \sqrt{2 \pi}} \cdot \exp \left(-\frac{\left(\theta-\theta_{B}\right)^{2}}{2 \sigma^{2}}\right)
$$

Where, $\sigma^{2}$ is the angular dispersion of thermal fluctuations of the magnetic moment angle of a carbon atom. To evaluate this dispersion, the potential energy change of the magnetic moment under the influence of the thermal energy should be evaluated:

$$
\mu_{C} B\left(1-\cos \theta_{T}\right) \approx k_{B} T_{C V D}
$$

Where, $\mu_{C}$ is the induced magnetic moment of a carbon atom $\mu_{C}=1.25 \mu_{B}$ (evaluations in reference [34], $\mu_{B}=5.788 \cdot 10^{-5} \mathrm{eV} / T, \mathrm{~B}$ is the magnetic induction of the catalyst surface, $\theta_{T}=\theta-\theta_{B} T_{C V D}$ is the operating temperature of the CVD process, $k_{B}=8,6173324(78) \cdot 10^{-5} \mathrm{eV} / \mathrm{K}$ is the Boltzmann constant. Hence,

$$
2 \sin ^{2} \frac{\theta_{T}}{2} \approx \frac{k_{B} T_{C V D}}{\mu_{C} B} .
$$

Taking into consideration one of the main problems of the nanotubes growth control - the chirality control - it is necessary to seek for the small fluctuation angle $\theta$. Then

$$
\sigma^{2}=\theta_{T}^{2}=\frac{2 k_{B} T_{C V D}}{\mu_{C} B} .
$$

The condition of the small fluctuation angle (e.g., $<10^{\circ}$ ) at a certain temperature of the CVD process imposes limitations on the values of the demanded magnetic induction $B$.

Taking into account the ratio between the 
chirality angle and the direction of the magnetic field $\phi=\theta_{B}$, Fig. 10 displays the predictable scattering of chiralities for nanotubes of approximately the same diameter.

We are also able to evaluate the necessary value of the magnetic field $\mathrm{B}$ providing the expected chirality angles scattering, e.g. $\sigma=0.2$ (approximately, $12^{\circ}$ ) leads to the $\mathrm{B}$ evaluation for the CVD process temperature $\quad T_{C V D}=700^{\circ} \mathrm{C}$ as $B=\frac{2 k_{B} T_{C V D}}{\sigma^{2} \mu_{C}} \approx \frac{16755}{\sigma^{2} \mu_{C}} \approx 57895 \approx 6 \cdot 10^{4}$ T. For small angle dispersions (Eq. 4) the high local magnetic field on the nanoparticle surface is necessary. The result also strongly depends on the carbon atom magnetic moment $\mu_{C}$.

Speaking about the possible errors in diameters of growing nanotubes, their evaluation from beneath is defined by the minimal variations in parameters of the chirality vector $\vec{c}=(n, m) \Delta n$ and $\Delta m$, which are equal.

Taking into consideration the formula for calculating the diameter of CNT:

$$
d=\frac{\sqrt{3} a}{\pi} \sqrt{m^{2}+n^{2}+m n}
$$

where, $\alpha=0.142 \mathrm{~nm}$ is the distance between the neighbouring carbon atoms in the graphite plane.

The relationship between chirality indices ( $n$ and $m$ ) and the angle $\phi$ is given by the ratio:

$$
\sin \phi=\frac{m \sqrt{3}}{2 \sqrt{m^{2}+n^{2}+m n}} .
$$

Then the minimum relative error in the diameter of CNT is possible to define as:

$$
\varepsilon_{d}=\frac{\Delta d}{d} \approx \frac{\sqrt{5 m^{2}+5 n^{2}+8 m n}}{2\left(n^{2}+m^{2}+m n\right)} .
$$

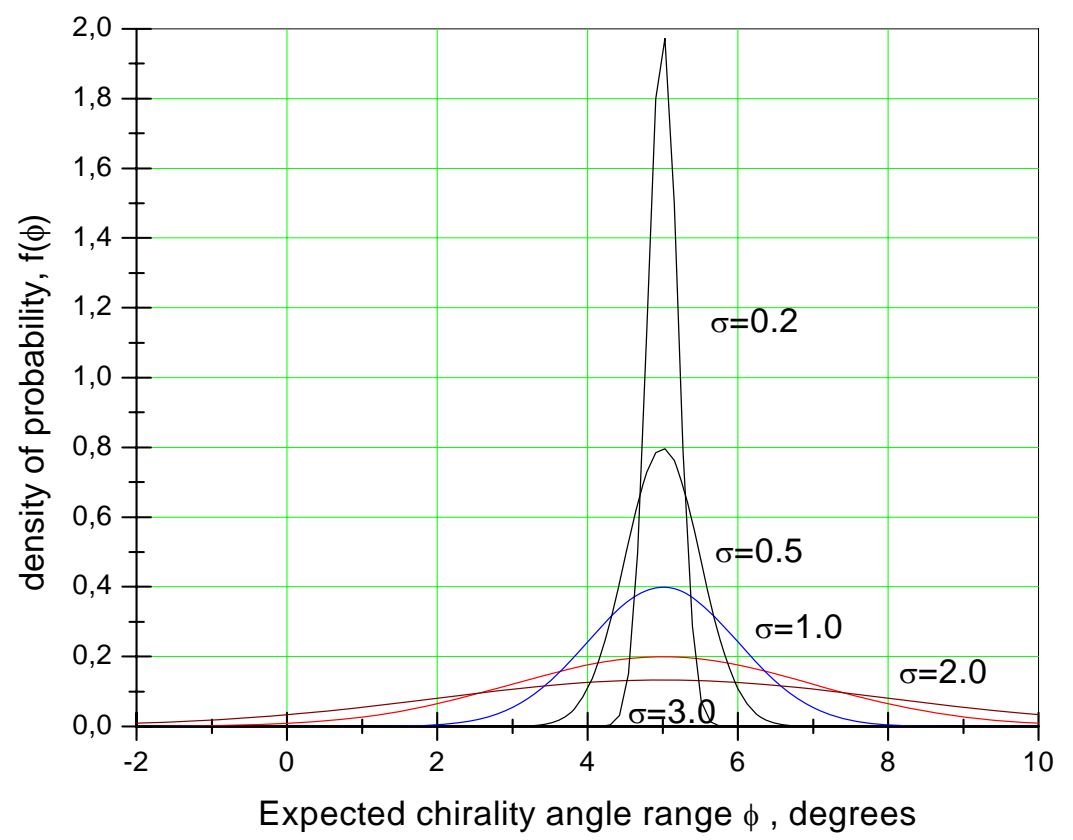

Fig. 9 The predictable scattering of chiralities for nanotubes of approximately similar diameters.

Particularly, in the case of arm-chair CNT $(m=0)_{\varepsilon_{d}} \approx \frac{\sqrt{5}}{2 n}$, in the case of zig-zag CNT $(m=n)$ $\varepsilon_{d} \approx \frac{1}{2 \sqrt{2} n}$.

Thus, the errors in the diameter of growing CNTs are incorporated in discrete morphological properties. However, these minimum estimates are only reinforced, given the obvious errors in the size of catalyst nanoparticles are taken into consideration.

\section{Magnetoresistance Phenomenon for Nanomemory Devices}

The GMR (Giant magnetoresistance) was discovered in 1988 as a large change in resistance of 


\section{Electromagnetic Simulations and Magnetically Stimulated Nanotube Growth}

magnetic $\mathrm{Fe} / \mathrm{Cr}$ multilayer in the presence of an applied magnetic field [37]. Soon after GMR was discovered in $\mathrm{Fe} / \mathrm{Cr} / \mathrm{Fe}$ trilayers [38]. As it was shown later, the effect can be obtained in trilayers having other magnetic materials such as Co [39].

Such trilayer structures, i.e. sandwiches of two ferromagnetic metals separated by a thin spacer layer of normal metal (Fig. 11) are of great industrial importance. They are called spin-valves and are used as magnetic field sensors. The resistance of the device is dependent on the relative magnetization orientation of the ferromagnets. $R_{p}$ is the resistance when the magnetizations are parallel and $\mathrm{R}_{\mathrm{A}}$ is the resistance whe they are antiparallel. The GMR ratio is defined as

$$
\mathrm{GNR}=\frac{\mathrm{R}_{\mathrm{A}}-\mathrm{R}_{\mathrm{P}}}{\mathrm{R}_{\mathrm{P}}} .
$$

TMR (Tunnelling magneto resistance) was discovered in 1975 by M. Jullière [40] in a device that consisted of two Fe films separated by Ge. It was first in 1995 when room temperature TMR was discovered by J. S. Moodera [41] and caused a great interest in spintronics after GMR was discovered in 1988 [37]. Our idea is that the same effect can be reached by introducing into the $\mathrm{N}$ space semiconductor CNTs.

The TMR signal operates in the same way as the GMR:

$$
\mathrm{TNR}=\frac{\mathrm{R}_{\mathrm{A}}-\mathrm{R}_{\mathrm{P}}}{\mathrm{R}_{\mathrm{P}}}
$$

where, $R_{P}\left(=1 / G_{P}\right)$ and $R_{A}\left(=1 / G_{A}\right)$ are the resistances (conductances) of the device for parallel and antiparallel orientations respectively of the ferromagnets magnetization. However, the nature of TMR is different than that of the GMR.

The resistance for the antiparallel magnetization is normally higher than for the parallel one. At the applied zero magnetic field, the relative orientation of the magnetization is governed by the exchange coupling between the ferromagnetic layers. The sign and size of the interlayer exchange coupling is dependent on the thickness of the nonmagnetic spacer. The coupling can thus be ferromagnetic and antiferromagnetic dependent on the spacer thickness $[42,43]$.

The device shown in Fig. 12 is the so-called CPP (Current perpendicular to the plane) geometry. The resistance of such geometry is very low and difficult to detect. For practical applications, structures with the CIP (Current in the plane) are used because they have higher resistance and thus higher difference with the magnetic field [39].

The GMR can be understood through the Mott's two-current model [44]. According to that model, the electrical conductivity of metal can be described by two more or less independent channels, one for majority spins and the other for minority spins. Scattering processes that conserve spin states are much more probable than the processes that flip spins.

Another view proposed by Mott is that the scattering probability of spin up and spin down is quite different, independent on the nature of the scattering process [45]. This is shown schematically in Fig. 10.

The difference in the resistance in ferromagnets can be explained by the exchange split band structure. The scattering of the electrons depends on where the electron band crosses the Fermi [39].

As mentioned above, commercial spin-valves have normally the CIP (Current in plane) geometry. This gives higher resistance and thus higher resistance difference between parallel and antiparallel spin orientations. Having the current in plane of the sandwich will qualitatively give the same effect as in 

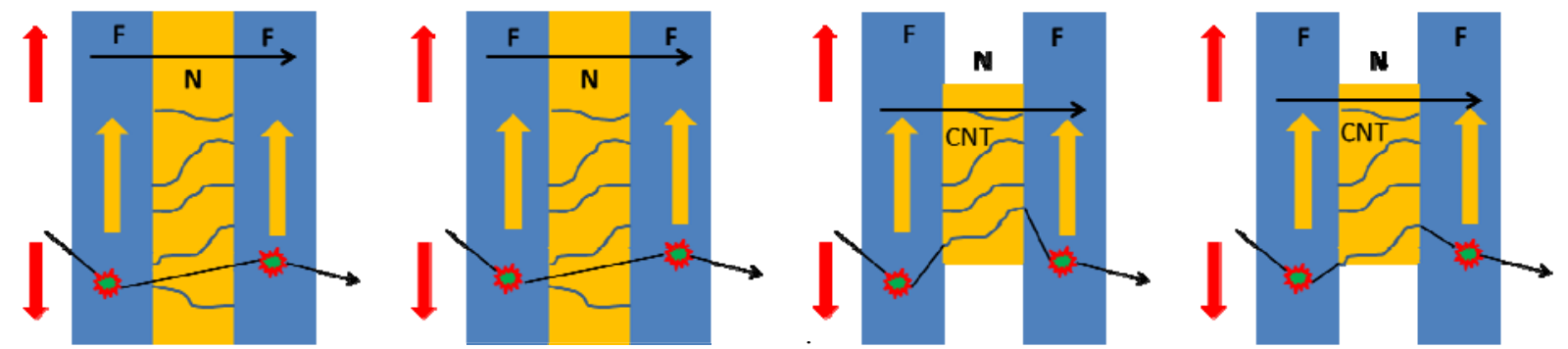

Fig. 10 GMR device, a thin normal metal spacer $(\mathrm{N})$ separates two ferromagnets $(\mathrm{F})$, the current flows perpendicular to the plane of the sample, $\mathrm{N}$ space can be filled by introduced (or grown) CNT, e.g. - metal-like.
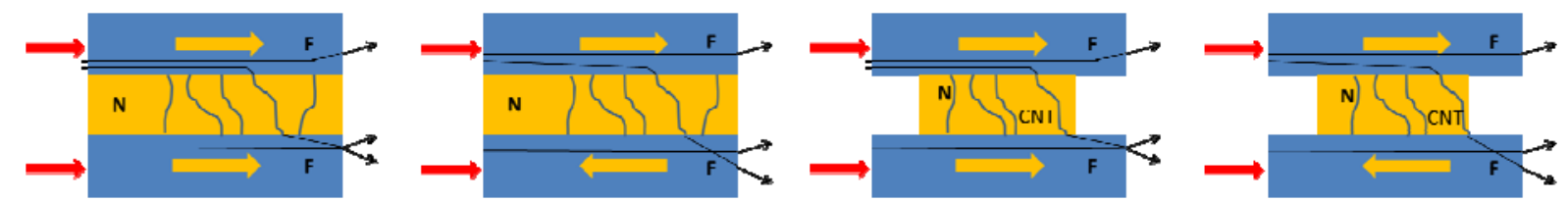

Fig. 11 CIP spin-valve and equivalent resistor mode, separate channels are for minority and majority spins, the electrons scatter from one $F$ layer to the other on the way through the sandwich.

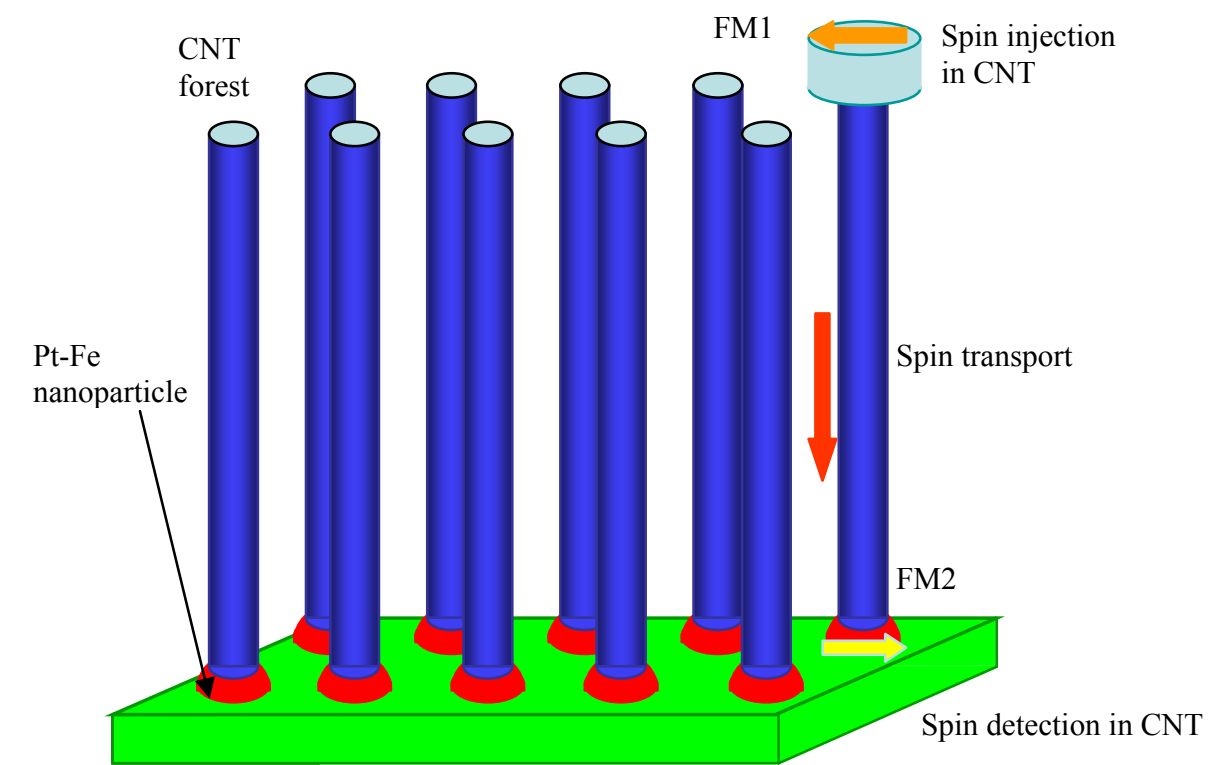

Fig. 12 CNTs forest is grown on the Fe-Pt nanoparticles of the predefined radius as a fragment of magnetic nanomemory device, which can be realized if spin injection, spin transport and spin detection (spin recording) are provided.

CPP devices. This situation is shown schematically in Fig. 12. Electrons with minority and majority spins are treated separately. When the electrons flow through the sandwich they will scatter back and forth from the upper F layer to the lower one.

The perfect picture of the magnetically stimulated CVD process for growing CNTs can be presented as a CNT forest (Fig. 13). Such a system of nanotubes can also be considered as a prototype of the magnetic memory, where ferromagnetic nanoparticles serve as cells of the magnetic memory - that is, ferromagnetic contacts are controlled by spin pulses, the transport of which is provided by nanotubes.

\section{Conclusions}

Electromagnetic properties of $\mathrm{Pt}$ - and Fe-CNT interconnects are considered from the point of view of mechanical stability and electrical efficiency. CNT-Fe interconnects are stronger mechanically. However, CNT-Pt interconnects having smaller resistances are more suitable electrically and more effective for various electronic nanodevices. 
The use of Pt-Fe nanoclusters during the CVD magnetically stimulated growth allows controlling with some limitation the diameter and chirality of growing CNTs. The diameter of nanoclusters defines the diameter of CNTs, while the angles of chiralities are correlated with the orientation of external permanent magnetic field.

Pt-Fe nanoclusters and CNTs composition is the prototype of the magnetic nanomemory devices. The magnetic efficiency depends on the stoichiometry coefficient $\mathrm{x}$ and the ordering of Pt-Fe atomic structure.

Chemically ordered Fe-Pt nanoparticles, where we meet the sequence of $\mathrm{Pt}$ and Fe layers, allows us to provide all possible predicted magnetic properties. In case of substitutional disorder, this advantage disappears and we should talk about the percolation phenomenon of $\mathrm{Fe}_{\mathrm{x}} \mathrm{Pt}_{1-\mathrm{x}}$ ferromagnetism via $\mathrm{x}$ alteration.

The CVD process of CNTs growth with the presence of Fe-Pt nanoparticles in the conditions of a strong magnetic field is a more orderly process.

Statistical dispersion of CNTs output according to the diameter is determined by a discrete number of effective bonds (especially in the case of small diameters of growing CNTs).

Here with the dependence of the number of the effective bonds, formed on the nanoparticle perimeter, on the chirality angle is the essential tool for the morphology control of the future CNT.

The balance factor of the thermal energy and magnetic energy in deposited nanoparticles of carbon atoms plays the decisive role in evaluating the dispersion of chirality angles in CNTs.

The morphology of the forthcoming growing CNT stimulated by the magnetic field is set when forming the perimeter of the CNT base and the character of the magnetic orientation in effective bonds.

We can consider CNT forest magnetically stimulated by the CVD process as a fragment of the magnetic nanomemory. CNT chirality in this case defines the efficiency of magnetic cells access (rates of exchange).

\section{Acknowledgments}

This study was supported by Grant EU FP7 CACOMEL project FP7-247007, Call ID FP7-PEOPLE-2009-IRSES，2010-2014 Nanocarbon based components and materials for high frequency electronics. Evaluating the contribution of the research team members we should point out that V I Gopeyenko and $\mathrm{N} \mathrm{Yu}$ Burlutskaya made a large amount of calculations, Yu F Zhukovskii and F Micciulla made the critical analysis of CVD details of CNTs growth, Yu N Shunin, T Lobanova-Shunina, and S Bellucci made the review of theoretical approaches and wrote most of the manuscript. We also thank Prof. E A Kotomin and Prof. S A Maksimenko for stimulating discussions on the topic.

\section{References}

[1] Shunin, Yu. and Kiv, A., Eds. 2012. Nanodevices and Nanomaterials for Ecological Security, Series. Nato Science for Peace Series B - Physics and Biophysics. Hiedelberg: Springer Verlag, 363.

[2] Shunin, Yu. N., Zhukovskii, Yu. F., Burlutskaya, N. Yu., Gopejenko, V. I. and Bellucci, S. 2012. Nanodevices and Nanomaterials for Ecological Security, Series: Nato Science for Peace Series B - Physics and Biophysics. Edited by Shunin, Yu., and Kiv, A. Hiedelberg: Springer Verlag, 237-62.

[3] Shunin, Yu. N., Zhukovskii, Yu. F., Gopejenko, V. I., Burlutskaya, N., Lobanova-Shunina, T. and Bellucci, S. 2012. Journal of Nanophotonics 6 (1): 061706-1-16.

[4] Shunin, Yu. N. and Schwartz, K. K. 1997. Computer Modelling of Electronic and Atomic Processes in Solids. Edited by Tennyson, R. C., and Kiv, A. E. Dodrecht/Boston/London: Kluwer Acad. Publisher, 241-57.

[5] Shunin, Yu. N., Zhukovskii, Yu. F., Gopejenko, V. I., Burlutskaya, N. and Bellucci, S. 2011. Nanoscience and Nanotechnology Letters 3: 1-10.

[6] Economou, E. L. 2006. Green's Functions in Quantum Physics Solid State; Ser. 7. Berlin/Heidelberg: Springer Verlag.

[7] Ziman, J. M. 1979. Models of Disorder. New York-London: Cambridge Univ. Press, Ch. 10. 
[8] Shunin, Yu. N., Zhukovskii, Yu. F., Burlutskaya, N. and Bellucci, S. 2012. Journal of Nanoelectronics and Optoelectronics 7 (1): 3-11.

[9] Capobianchi, A., Colapietro, M., Fiorani, D., Foglia, S., Imperatori, P., Laureti, S. et al. 2009. Chem. Mater. 21 (10): 2007-9.

[10] Capobianchi, A., Campi, G., Camalli, M. and Veroli, C. Z. 2009. Kristallogr 224 (8): 384-8.

[11] Faustini, M., Capobianchi, A., Varvaro, G. and Grosso, D. 2012. Chem. Mater. 24: 1072-9.

[12] Bellucci, S. 2005. Physica Status Solidi (c) 2 (1): 34-47.

[13] Moisala, A., Nasibulin, A. G. and Kauppinen, E. I. J. 2003. Phys. Condens. Mater. 15: S3011-35.

[14] Meyyappan, M., Ed. 2005. Carbon Nanotubes Science and Applications. Florida: CRC Press LLC Google e-Book.

[15] Muller, T. E., Reid, D. G., Hsu, W. K., Hare, J. P., Kroto, H. W. and Walton, D. R. et al.. 1997. Carbon 35: 951-61.

[16] Xie, S., Chang, B., Li, W., Pan, Z., Sun, L., Mao, J., and et al. 1999. Adv. Mater. 11: 1135.

[17] Cassell, A. M., Raymakers, J. A., Kong, J. and Dai, H. J. 1999. Phys. Chem. B 103: 6484.

[18] Su, M., Zheng, B. and Liu, J. 2000. Chem. Phys. Lett. 322: 32.

[19] Su, M., Li, Y., Maynor, B., Buldam, A., Lu, J. P. and Liu, J. J. 2000. Phys. Chem. B 104: 6505-12.

[20] Fan, S. S., Chapline, M. G., Franklin, N. R., Tombler, T. W., Cassell, A. M. and Dai, H. 1999. Science 283: 512.

[21] Cheng, H. M., Li, F., Su, G., Pan, H. Y., He, L. L., Sun, X., and et al. 1998. Appl. Phys. Lett. 72: 3282.

[22] Li, Y. and Liu, J. 2001. Chem. Mater. 13: 1008-14.

[23] Lee, D. C., Mikulec, F. V., and Korgel, B. A. 2004. Amer. Chem. Soc. 126: 4951.

[24] Chen, G. Y., Jensen, B., Stolojan, V. and Silva, S. R. P. 2011. Carbon: 49 (1): 280-5.

[25] Shang, N. G., Tan, Y. Y., Stolojan, V., Papakonstantinou, P. and Silva, S. R. P. 2010. Nanotechnology 21: 505604.

[26] Kumar, M. and Ando, Yo. 2010. Journal of Nanoscience and Nanotechnology 10: 3739.

[27] Takagi, D., Hibino, H., Suzuki, S., Kobayashi, Y. and
Homma, Y. 2007. Nano Lett. 7: 2272.

[28] Takagi, D., Homma, Y., Hibino, H., Suzuki, S. and Kobayashi, Y. 2006. Nano Lett. 6: 2642.

[29] Liu, H., Takagi, D., Ohno, H., Chiashi, S. Chokan, T. and Homma, Y. 2008. Appl. Phys. Express 1: 014001.

[30] Bellucci, S., Zhukovskii, Yu. F., Gopejenko V. I., Burlutskaya, N. and Shunin, Yu. N. 2012. CIMTEC2012 Theses, June 10-14 Montecatini, Terme, Tuscany, Italy, 78.

[31] Sun, A. C., Kuo, P. C., Chen, S. C., Chou, C. Y., Huang, H. L. and Hsu, J. H. J. 2004. Appl. Phys. 95 (11): 7264.

[32] Elkins, K., Li, D., Poudyal, N., Nandwana, V., Jin, Zh., Chen, K. and et al. 2005. J. Phys. D 38: 2306.

[33] Yan, M. L., Sabirianov, R. F., Xu, Y. F., Li, X. Z. and Sellmyer, D. J. 2004. IEEE Trans. on Magnetics 40 (4): 2470.

[34] Kim, Y.-H., Choi, J. and Chang, K. J. 2003. Phys. Rev. B 68: 125420 .

[35] Medwal, R., Sehdev, N. and Annapoorni, S. J. 2012. Phys. D Appl. Phys. 45: 055001.

[36] Arabshahi, H., Hematabadi, A., Bakhshayeshi, A. and Ghazi, M. 2012. World Applied Programming 2 (8): 415-20.

[37] Baibich, M. N., Broto, J. M., Fert, A., Van Dau, F. N., Petroff, F., Eitenne, P. and et al. 1988. Phys. Rev. Lett. 61: 2472.

[38] Binasch, G., Grünberg, P., Saurenbach, F. and Zinn, W. 1989. Phys. Rev. B 39: 4828.

[39] Tsymbal, E. Y. and Pettifor D. G. 2001. Solid State Physics 56: 113.

[40] Julliére, M. 1975. Phys. Lett. A 54: 225.

[41] Moodera, J. S., Kinder L. R., Wong T. M. and Meservey R. 1995. Phys. Rev. Lett. 74: 3273.

[42] Parkin, S. S. P., More N. and Roche K. P. 1990. Phys. Rev. Lett. 64: 2304.

[43] Parkin, S. S. P., Bhadra R. and Roche K. P. 1991. Phys. Rev. Lett. 66: 2152.

[44] Mott, N. 1936. Proc. Royal. Soc. 153: 699.

[45] Mott, N. F. 1936. Proc. Royal. Soc. 156: 368. 